\title{
Stage-specific Activity of Potential Antimalarial Compounds Measured in Vitro by Flow Cytometry in Comparison to Optical Microscopy and Hypoxanthine Uptake
}

\author{
Carmen E Contreras/ ${ }^{+}$, María A Rivas, José Domínguez* , Jaime Charris*, Mario Palacios, \\ Nicolás E Bianco, Isaac Blanca
}

Instituto de Inmunología, Facultad de Medicina *Laboratorio de Síntesis Orgánica, Facultad de Farmacia, Universidad Central, Apartado Postal 50109, Caracas 1050 A, Venezuela

The evaluation of new antimalarial agents using older methods of monitoring sensitivity to antimalarial drugs are laborious and poorly suited to discriminate stage-specific activity. We used flow cytometry to study the effect of established antimalarial compounds, cysteine protease inhibitors, and a quinolone against asexual stages of Plasmodium falciparum. Cultured $\mathrm{P}$. falciparum parasites were treated for $48 \mathrm{~h}$ with different drug concentrations and the parasitemia was determined by flow cytometry methods after DNA staining with propidium iodide. P. falciparum erythrocytic life cycle stages were readily distinguished by flow cytometry. Activities of established and new antimalarial compounds measured by flow cytometry were equivalent to results obtained with microscopy and metabolite uptake assays. The antimalarial activity of all compounds was higher against P. falciparum trophozoite stages. Advantages of flow cytometry analysis over traditional assays included higher throughput for data collection, insight into the stage-specificity of antimalarial activity avoiding use of radioactive isotopes.

Key words: Plasmodium falciparum - antimalarial compounds - flow cytometry - hypoxanthine uptake - chloroquine - quinolone

Drug resistance of Plasmodium falciparum, the most deadly human malaria parasite, is a major factor in the widespread persistence of malaria (Ouellette \& Kunding 1997, Macreadi et al. 2000). Current efforts focus on research into novel compounds and on measures to prevent or delay resistance once new drugs are introduced. However, malaria therapy has generally not taken into consideration the stage-specificity of action of different drugs. This is an important consideration, since inappropriate timing of administration of antimalarial drugs might limit drug efficacy and favor the selection of drug-resistant parasites.

Few studies have focused on the in vitro stage-specific efficacy of antimalarial compounds (Chimanuka et al. 2001). Most in vitro studies monitoring resistance and susceptibility to antimalarial compounds have been performed by microscopy and by uptake of a radiolabelled nucleic acid precursor ${ }^{3}[\mathrm{H}]$-hypoxanthine (Desjardins et al. 1979). They are poorly suited to discriminate stagespecific activity. More recently, flow cytometry analysis (FCA) using different fluorescent dyes has been reported (van Vianen et al. 1990, Pattanapanyasat et al. 1996, 1997).

We used propidium iodide (PI) whose incorporation as fluorescent molecule has been shown to be proportional to the DNA parasite content. In the present study, results of in vitro effect of antimalarial compounds on $P$. falciparum parasitized erythrocytes obtained from FCA

Financial support: Conicit, grant S1-97001305

${ }^{+}$Corresponding author. Fax: +58-212-693.2815. E-mail: contrerc@camelot.rect.ucv.ve

Received 14 July 2003

Accepted 18 February 2004 were compared with other assays. FCA results were comparable to optical microscopy observation and to $\left[{ }^{3} \mathrm{H}\right]-$ hypoxanthine uptake assay. Moreover, FCA offers advantages of high throughput, ready automation, simplified data processing, and easy determination of the stage-specific effectiveness of potential antimalarial compounds.

\section{MATERIALS AND METHODS}

Parasites - Chloroquine sensitive NF54 (kindly provided by Dr T Shapiro, Johns Hopkins University, Baltimore, $\mathrm{MD}$ ) and $\mathrm{FCB}_{2}$ strains, and a chloroquine resistant FCB strain (MR4 Resource Center, ATCC), as well as a Venezuelan (VEN, unknown chloroquine sensitivity) $P$. falciparum strains were used in this study. All strains were maintained in continuous culture as previously described (Trager \& Jensen 1976) at 2.4\% hematocrit using type 0+ human erythrocytes in RPMI 1640 medium supplemented with $2 \mathrm{mM} \mathrm{L}$-glutamine (Gibco, Grand Island, NY), $10 \%$ heat inactivated pooled $\mathrm{AB}+$ human serum (University Hospital Blood Bank, Caracas, Venezuela), $25 \mathrm{mM}$ HEPES (Calbiochem, San Diego, CA), and 5\% $\mathrm{NaHCO}_{3}$ under a $3 \% \mathrm{O}_{2}, 4 \% \mathrm{CO}_{2}$, and $93 \% \mathrm{~N}_{2}$ gas mixture. Parasite synchronization was performed by sorbitol treatment (DSorbitol, Sigma) as previously described (Lambros \& Vanderberg 1979). Viability and parasitemia of cultured parasites were calculated by light microscopy analysis of blood smear stained with Giemsa (5000 erythrocytes counted per blood smear).

Drugs - The quinolone derivative $3\left(\mathrm{C}_{16} \mathrm{H}_{10} \mathrm{Cl}_{2} \mathrm{~N}_{4} \mathrm{O}\right)$ ( $\mathrm{R}$ 2,4-Cl) which lacks hydrogen-bonds (Dominguez et al. 1998) was synthesized with high yields from the intermediate 2 methylthio-3-ciano-4-quinolones by treatment with hydrazine hydrate in refluxing pyridine and guanidine hydrochloride with potassium carbonate in refluxing DMF (Hooper \& Wolfson 1985, Tripathi et al. 1993, Dominguez 
et al. 1996). Quinolone 3 (purity assessed by HPLC was > 99\%) previously showed antimalarial activity (Dominguez et al. 1996). Vinyl sulfone (Mu-Leu-Hph-VSPh), and fluoromethyl ketone (Mu-Phe-Hph- $\mathrm{CH}_{2} \mathrm{~F}$ ) protease inhibitors (Rosenthal et al. 1996) were kindly provided by Dr P Rosenthal, San Francisco General Hospital, University of California, CA; chloroquine (Sigma, St. Louis, MO) or artemisinin (Aldrich Chemical CO, Milwaukee, WI) were also included in this study as controls.

Drug susceptibility assays - Different techniques were used to measure antimalarial activity of compounds included in this study, FCA of PI incorporation into parasite DNA, $\left[{ }^{3} \mathrm{H}\right]$-hypoxanthine uptake, and parasitemia by light microscopy. Antimalarial activity was performed in three parallel microtiter plate wells (Costar, Corning, NY, 96 flatbottom plates). Triplicate wells containing $100 \mu \mathrm{l}$ of drug were mixed with $100 \mu \mathrm{l}$ of $P$. falciparum parasitized erythrocytes ( $0.25 \%$ parasitemia, and $2.4 \%$ hematocrit). Quadruplicates of three different controls were included in each microplate: nonparasitized human erythrocytes (negative control), $P$. falciparum parasitized human erythrocytes without drug, and P. falciparum parasitized human erythrocytes without drug in the presence of $0.2 \%$ DMSO (positive control). Stock solutions of HPLC-purified or recrystallized compound were dissolved in either DMSO (quinolone 3, protease inhibitors, and artemisinin) or distilled water (chloroquine). DMSO soluble drugs were later diluted 500 folds in medium, and finally serially diluted in $0.2 \%$ DMSO-medium (to maintain constant solvent concentration). Dose-response curves of each drug included 10 concentrations of 1.8 fold dilutions yielding final concentrations between $0.16-2500 \mathrm{ng} / \mathrm{ml}$. Final concentrations were expressed in $\mathrm{nM}$.

Calculation of $I_{50}-\mathrm{IC}_{50}$ was calculated from each drug dose-response curve after logarithmic transformation and fit to a generalized sigmoidal function by means of the Marquardt algorithm (Bard 1974), and analyzed for goodness of fit ( $r^{2}$ value).

DNA staining and flow cytometry analysis - Drug activity against malarial parasites was determined by measuring incorporation of PI (Sigma) as previously described (Pattanapanyasat et al. 1993, 1997) with some modifications. After $48 \mathrm{~h}$ incubation, drug-treated and control parasitized erythrocytes, were washed twice with PBS by centrifugation each time at $200 \mathrm{x} \mathrm{g}$, and fixed overnight with $0.25 \% \mathrm{v} / \mathrm{v}$ glutaraldehyde (Sigma) in PBS. After washing with PBS, fixed cells were mixed with a recently prepared and filtered $\mathrm{PI} / \mathrm{H}_{2} \mathrm{O}$ solution (final concentration $50 \mu \mathrm{g}$ / $\mathrm{ml}$ ) in Tris $1 \mathrm{M}, \mathrm{pH} 8$ containing RNAse, Nonidet P-40 and $\mathrm{NaCl}$ as described by Vindelov (1977), and incubated for 4 $\mathrm{h}$ in dark at $37^{\circ} \mathrm{C}$. Cells were vigorously mixed and intracellular parasite DNA content was determined by PI incorporation using a flow cytometer (Epics-Elite, BeckmanCoulter, Miami, FL) equipped with a Enterprise Argon-ion laser tuned at $488 \mathrm{~nm}$. PI red fluorescence intensity was collected through a $575 \mathrm{~nm}$ band pass filter. List mode data from 100,000 erythrocytes was stored and processed for each well in an Elite-ESP Software (Coulter). Results were reported as DNA stained cells (\%).

Optimal FCA conditions were initially established using chicken erythrocytes since they are nucleated cells able to incorporate PI in their DNA and localized differently than the normal human erythrocytes in flow cytometry histograms. Suspensions of uninfected human erythrocytes/chicken erythrocytes $\left(2.5 \times 10^{6}\right.$ normal human erythrocytes/250-2.5 × $10^{5}$ chicken erythrocytes) yielded histograms which clearly differentiated both populations from contaminating human peripheral blood leukocytes, platelets, or reticulocytes (data not shown). Such contaminating cells were subsequently eliminated by extensive washing of uninfected human erythrocyte suspensions. In initial trials, a PI concentration of $50 \mu \mathrm{g} / \mathrm{ml}$ and $4 \mathrm{~h}$ incubation at $37^{\circ} \mathrm{C}$ in the dark was optimal to discriminate among parasitized and non parasitized erythrocytes and other nucleated cells.

Tritiated hypoxanthine uptake - Incorporation of ${ }^{3}[\mathrm{H}]$ hypoxanthine was performed as previously described (Desjardins et al. 1979) with modifications (Posner et al. 1997). Briefly, nonparasitized and parasitized erythrocytes (with or without drug) were incubated for $24 \mathrm{~h}$ prior to addition of $2 \mu \mathrm{Ci} /$ well of $\left[{ }^{3} \mathrm{H}\right]$-hypoxanthine $[1 \mathrm{mCi} / \mathrm{ml}$ ethanol-water (1:1) solution, Amersham, Pharmacia, England] diluted with medium. After an additional $18 \mathrm{~h} \mathrm{incu-}$ bation, cells were harvested (Skatron Instruments) onto glass fiber filters (Wallac, TurKu, Finland) which were exhaustively washed. Radioactivity from dry filters was determined with a scintillation counter (LKB, Stockholm, Sweden).

Microscopic observation - Thin smears were made from nonparasitized and parasitized erythrocytes (with or without drug) after $24-48 \mathrm{~h}$ incubation at $37^{\circ} \mathrm{C}$. Parasitemias were calculated by counting the number of parasites per 5000 Giemsa-stained human erythrocytes using light microscopy.

\section{RESULTS}

P. falciparum life cycle stages can be distinguished by FCA - In order to determine the potential of flow cytometry to discriminate the stage of parasite infected erythrocytes, we first studied non-infected and asynchronous parasitized human erythrocytes stained with PI as described in Materials and Methods. Fig. 1A shows a representative fluorescent histogram trigger in red fluorescent light for non-parasitized human erythrocytes (HEn) used as negative control and parasitized human erythrocytes (HEp) whose parasitemia by light microscopy was $0.07 \%$ rings, $0.5 \%$ trophozoites, and $0.04 \%$ schizonts. Three different peaks of fluorescence intensity can be distinguished, indicative of different parasite DNA content or parasite stage. To determine whether these fluorescence peaks correspond to different parasite-stages, synchronized cultures of $P$. falciparum were prepared and submitted to FCA. Fig. 1B, shows the fluorescent histograms generated by each synchronized culture. Thus, overlay of the histograms shows that first peak is consistent with early ring (early rings ER, $16 \mathrm{~h}$ after merozoite invasion), and early trophozoite (early trophozoites ET, 16-22 $\mathrm{h}$ after merozoite invasion), while the second and third peaks were consistent with late trophozoite (late trophozoites LT, 22-30 $\mathrm{h}$ after merozoite invasion), and schizont (SCH 32-48 h after merozoite invasion) stages respectively. 

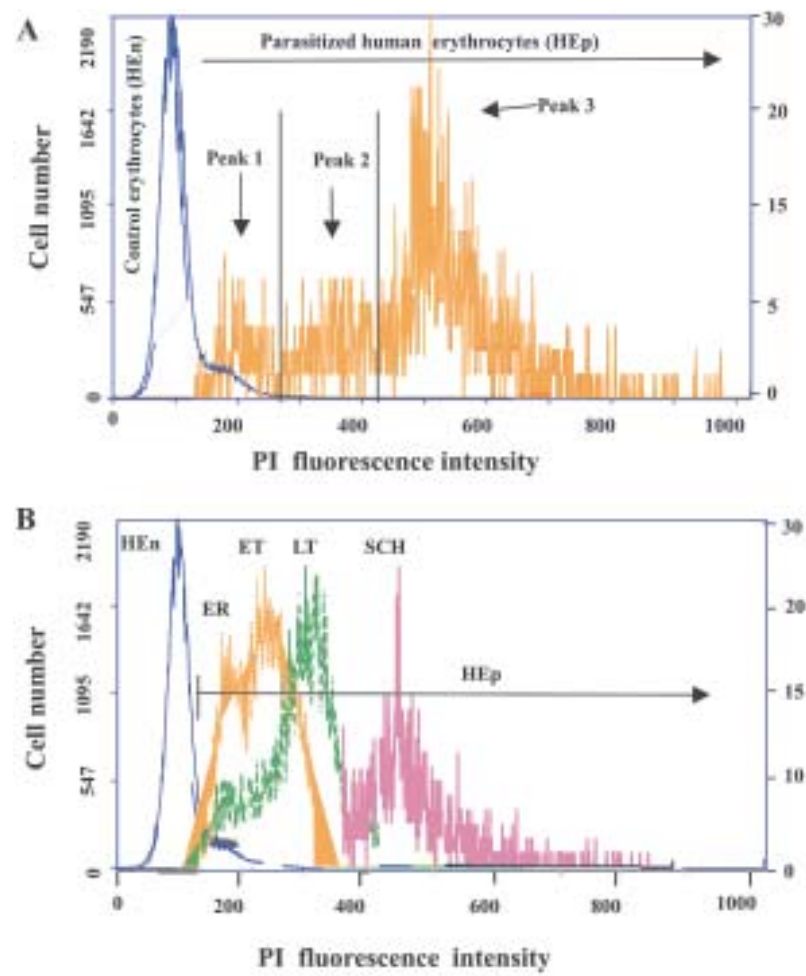

Fig. 1: flow cytometry assay of Plasmodium falciparum parasitized erythrocytes $\left(\mathrm{FCB}_{2}\right.$ strain) stained with propidium iodide (PI). A: overlap of typical histograms of HEn and HEp from asynchronous $P$. falciparum cultures gated on red fluorescent light. The scale of HEp fluorescent histogram was fixed 100 time lower to visualize the parasitized erythrocyte population. B: overlap of fluorescent histograms from synchronous $P$. falciparum cultures with ER (early rings), ET (early trophozoites), LT (late trophozoites), and $\mathrm{SCH}$ (schizonts).

Stage-specific efficacy of antimalarial compounds measured by FCA - To determine whether the efficacies of the compounds used in this study were stage specific, we first performed pilot experiments with asynchronous parasites treated with different drugs. Total and differential parasitemia were counted by optical microscopy and FCA as described above. The antimalarial activity of quinolone 3 was predominantly effective against trophozoite-stage parasites which showed $78 \%$ of parasitemia inhibition compared to that of the untreated parasites. Inhibition of parasitemia on ring and schizont stages was 33 and $46 \%$ respectively. To confirm this observation, we set up further experiments using synchronized parasites at different stages of their life cycle. Table I illustrates results obtained when P. falciparum parasitized erythrocytes were incubated (young rings: $16 \mathrm{~h}$, young trophozoites: $20 \mathrm{~h}$ ) with different concentrations of quinolone 3 and analyzed by FCA and optical microscopy. Similar results were observed with both techniques (Table I). A slight inhibition of ring-stage parasitemia (20\%) was observed only by FCA, at the highest quinolone 3 concentration $(1.4 \mu \mathrm{M})$. In contrast, again by FCA a significant inhibition of trophozoite-stage parasitemia of $80 \%$ was observed at $1.4 \mu \mathrm{M}$, and of $40 \%$ at $0.8 \mu \mathrm{M}$. A similar response was observed by optical microscopy where 88 and $50 \%$ inhibition were obtained at 1.4 and $0.8 \mu \mathrm{M}$ of quinolone 3 concentrations respectively.

Based on these results a dose-response curve was obtained for quinolone 3 using P. falciparum NF54 trophozoites. Fluorescent histograms were acquired for nonparasitized and parasitized erythrocytes incubated with increasing quinolone concentrations. As shown in Fig. 2A progressive drug effects were demonstrated as decreasing fluorescence. The antimalarial effective range of quinolone 3 was between 0.2 and $1.4 \mu \mathrm{M}$. We further tested the effect of other antimalarial compounds, two cysteine protease inhibitors, and artemisinin and chloroquine used as positive controls. As shown in Fig. 2B increasing drugeffects of fluoromethyl ketone (Mu-Phe- $\mathrm{Hph}-\mathrm{CH}_{2} \mathrm{~F}$ ) cysteine protease inhibitor was demonstrated as decreasing fluorescence. The antimalarial effect of this drug was between 0.5 and $250 \mathrm{nM}$. Similar results were obtained using another cysteine protease inhibitor such as vinyl sulfone (Mu-Leu-Hph-VSPh), as well as traditional drugs as chloroquine and artemisinin (data not shown).

Antimalarial $I C_{50}$ - Dose-response curves of some of the compounds studied by FCA indicate that trophozoite parasitemia inhibition is proportional to drug concentration. Fig. 3A shows representative dose-response curves of quinolone 3 and of two antimalarial drugs (chloroquine and artemisinin). An excellent fit of the data to the regression equation was obtained with each compound $\left(r^{2}: 0.999\right.$, data not shown). Antimalarials $\mathrm{IC}_{50}$ calculated from dose-response curves were consistent between tech-

TABLE I

Stage-specific antimalarial activity of quinolone 3 on synchronized Plasmodium falciparum parasites

\begin{tabular}{|c|c|c|c|c|c|c|c|c|}
\hline & \multicolumn{4}{|c|}{ Optical microscopy $a, b$} & \multicolumn{4}{|c|}{ Flow cytometry ${ }^{a, c}$} \\
\hline & Rings \% & Inhib \% & Troph \% & Inhib \% & Rings $\%$ & Inhib \% & Troph \% & Inhib \% \\
\hline HEn & & & & & $0.1 \pm 0.1$ & & $0.1 \pm 0.1$ & \\
\hline HEp & $0.2 \pm 0.03$ & & $0.8 \pm 0.2$ & & $0.45 \pm 0.1$ & & $0.5 \pm 0.1$ & \\
\hline $1.4 \mu \mathrm{M}$ & $0.3 \pm 0.04$ & 0 & $0.1 \pm 0.02$ & 88 & $0.4 \pm 0.1$ & 20 & $0.1 \pm 0$ & 80 \\
\hline $0.8 \mu \mathrm{M}$ & $0.2 \pm 0.04$ & 0 & $0.4 \pm 0.03$ & 50 & $0.5 \pm 0.1$ & 0 & $0.3 \pm 0.1$ & 40 \\
\hline $0.1 \mu \mathrm{M}$ & $0.3 \pm 0.03$ & 0 & $0.7 \pm 0.1$ & 13 & $0.5 \pm 0.1$ & 0 & $0.5 \pm 0.1$ & 0 \\
\hline
\end{tabular}

$a$ : synchronous parasites (NF54 strain) incubated with drug decreasing concentrations; $b$ : parasitemia, mean $\pm \mathrm{SD}, \mathrm{n}=3$ (Giemsa smears read by two persons); $c$ : parasitemia, mean $\pm \mathrm{SD}, \mathrm{n}=3$; Troph: trophozoites; Inhib: parasitemia inhibition; HEn: nonparasitized human erythrocytes; HEp: parasitized human erythrocytes 
A

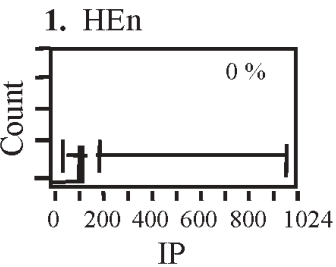

2. HEp

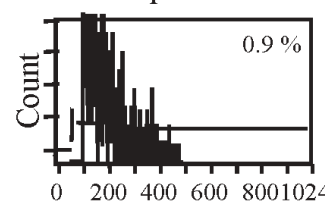

IP

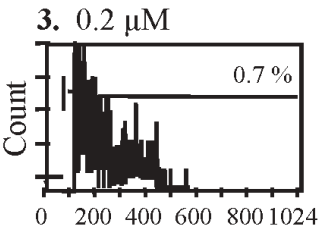

IP

B

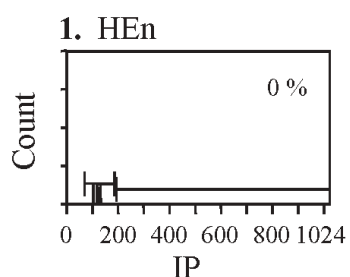

2. HEp

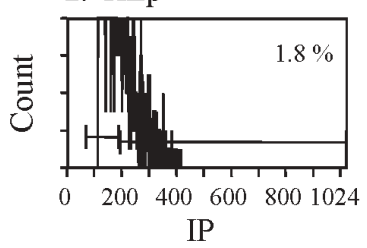

3. $0.5 \mathrm{nM}$

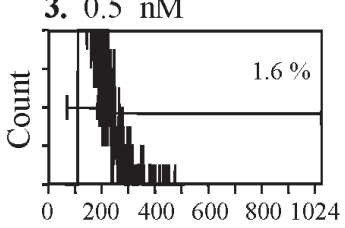

IP

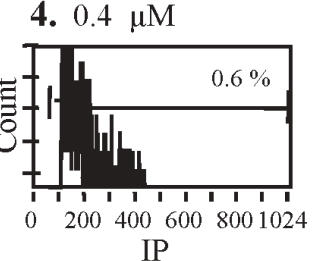

5. $0.8 \mu \mathrm{M}$

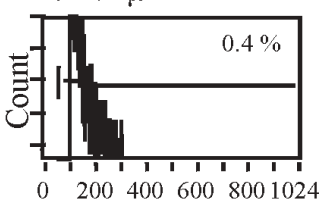

IP

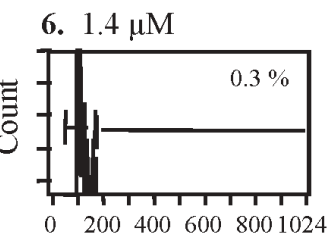

IP

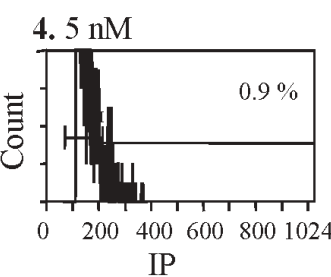

5. $50 \mathrm{nM}$

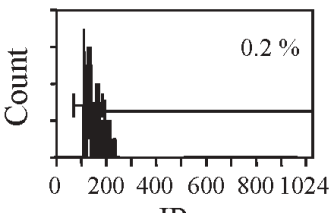

IP

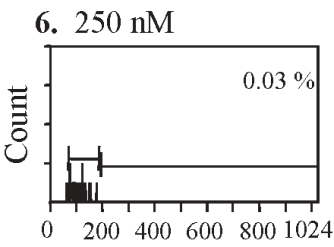

IP

Fig. 2: effect of quinolone 3 (A), and protease inhibitor Mu-PheHph- $\mathrm{CH}_{2} \mathrm{~F}$ (B) on Plasmodium falciparum synchronized trophozoites (VEN and NF54 strains respectively) measured by flow cytometry. Individual histograms show the $\%$ of parasitemia (FCA) corresponding to each drug concentration. Non-parasitized human erythrocytes (HEn) and parasitized human erythrocytes (HEp) without drug were included as negative and positive controls respectively.

niques and $P$. falciparum strains (data not shown). $\mathrm{IC}_{50}$ 's of $P$. falciparum VEN strain, were of $800 \mathrm{nM}$ for quinolone 3, $29 \mathrm{nM}$ for chloroquine, $8.5 \mathrm{nM}$ for artemisinin, $330 \mathrm{nM}$ for vinyl sulfone, and $15.7 \mathrm{nM}$ for fluoromethyl ketone cysteine protease inhibitors. As expected, chloroquine $\mathrm{IC}_{50}$ for the chloroquine-resistant strain $\mathrm{FCB}$, and for the chloroquine-sensitive strain NF54 were of $170 \mathrm{nM}$ and 10 $\mathrm{nM}$ respectively.

Comparison between $I_{50}$ 's of four antimalarial compounds measured by FCA, hypoxanthine uptake, and optical microscopy - Antimalarial activities of standard and new compounds measured by FCA were compared with those obtained by the hypoxanthine uptake assay, and optical microscopy, standard methods of assessing in vitro antimalarial drug activity. As depicted in Fig. 3B, a high correlation $\left(r^{2}: 0.999\right)$ was observed among $\mathrm{IC}_{50}$ calculated by FCA and hypoxanthine uptake. In addition, $\mathrm{IC}_{50}$ 's of FCB, VEN, and NF54 strains calculated from chloroquine optical microscopy dose-response curves were of $151 \mathrm{nM}, 33 \mathrm{nM}$, and $9 \mathrm{nM}$ respectively. Such $\mathrm{IC}_{50}$ 's were consistent with the ones calculated from FCA doseresponse curves which were of $170 \mathrm{nM}, 29 \mathrm{nM}$, and 10 $\mathrm{nM}$ respectively.

Correlation of antimalarial activity of quinolone measured by FCA and optical microscopy - Antimalarial activity of quinolone evaluated by FCA and optical microscopy was similar (Table II). Inhibitory growth effect was proportional to drug concentration after $48 \mathrm{~h}$ incubation. A significant correlation between both techniques was observed only at $0.8 \mu \mathrm{M}(\mathrm{p}<0.05)$. Quinolone activity at 2.5 and $0.8 \mu \mathrm{M}$ was evident since microscopy examination showed parasitemias of 0 and $0.4 \%$ respectively. Moreover, parasites look unhealthy with a compact chromatin and could be easily distinguished as dead parasites. In contrast, FCA does not detect morphological changes, indeed damaged or dead parasites may incorporate PI at their DNA as was demonstrated by the increased parasitemias observed $(0.2$ and $0.7 \%$ at 2.5 , and $0.8 \mu \mathrm{M}$ respectively).

\section{DISCUSSION}

FCA have proved to be useful to study diverse features related with human and experimental malaria (Janse et al. 1987, Pattanapanyasat et al. 1993, 1999, Kumaratilake \& Ferrante 2000, Saito-Ito et al. 2001).

TABLE II

Quinolone antimalarial activity correlation between flow cytometry and optical microscopy

\begin{tabular}{lccc}
\hline \multicolumn{4}{c}{ Parasitemia \% (Mean \pm SD) } \\
\hline $48 \mathrm{~h}$ & $\mathrm{OM}$ & FCA & $\mathrm{p}$ \\
\hline $\mathrm{HEp}$ & $2.3 \pm 0.1$ & $1.9 \pm 0.2$ & $\mathrm{NS}$ \\
$2.5 \mu \mathrm{M}$ & $0.0 \pm 0.0$ & $0.2 \pm 0.1$ & $\mathrm{NS}$ \\
$0.8 \mu \mathrm{M}$ & $0.4 \pm 0.0$ & $0.7 \pm 0.1$ & $<0.05$ \\
$0.1 \mu \mathrm{M}$ & $1.9 \pm 0.3$ & $1.6 \pm 0.4$ & $\mathrm{NS}$ \\
\hline
\end{tabular}

OM: optical microscopy; FCA: flow cytometry assay; HEp: parasitized human erythrocytes (Plasmodium falciparum NF54 strain) without drug; NS: not significant 

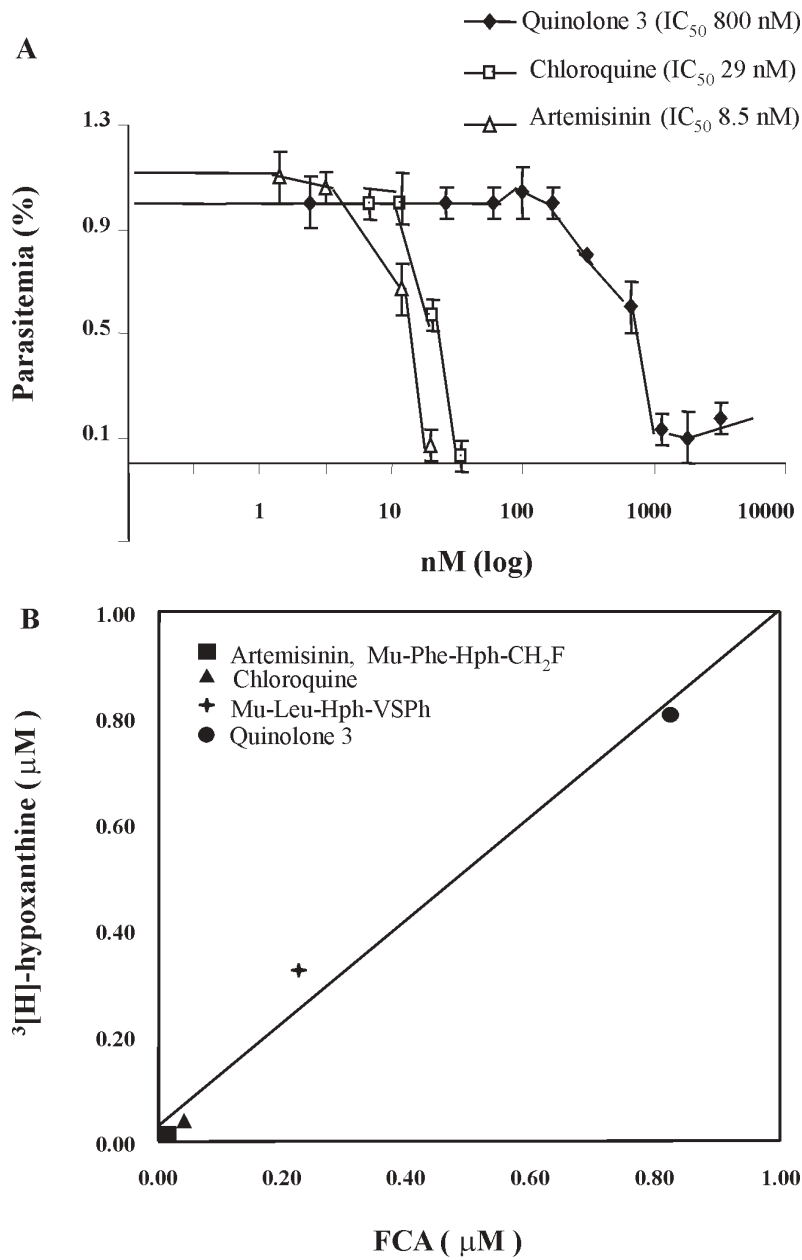

Fig. 3A: flow cytometry dose-response curves of quinolone 3, chloroquine and artemisinin on Plasmodium falciparum parasitized erythrocytes (VEN strain). Each point represents the mean \pm SD of triplicate measurements; $\mathrm{B}$ : correlation between $\mathrm{IC}_{50}$ 's of different compounds measured by flow cytometry and hypoxanthine uptake. Linear correlation, $r^{2}$ : 0.999; FCA: flow cytometry analysis

In this study, we have described by flow cytometry the anti-malarial activity of four drugs using continuos cultures of $P$. falciparum geographically different strains. Moreover, we compared the $\mathrm{IC}_{50}$ calculated by this method with those obtained by microscopy and ${ }^{3}[\mathrm{H}]$-hypoxanthine uptake.

Flow cytometry method enabled to discriminate three different populations of infected erythrocytes, corresponding to different asexual $P$. falciparum stages. Such populations can be electronically gated to determine the mean channel fluorescence intensity of PI uptake characteristic of each one. By monitoring the P. falciparum developmental stages and drug sensitivity, it was observed that the inhibitory effect of all tested antimalarial compounds was primarily directed to trophozoite stages, with little or not effect on ring stages.

These results agree with previous reports, showing that $P$. falciparum trophozoite stage was the most sensi- tive to in vitro treatment with chloroquine (Yayon et al. 1983, Cambie et al. 1991). Relevance of chronotherapy in malaria has been recently demonstrated in vivo studies of the rodent malaria parasite $P$. chabaudi chabaudi. This study indicates that the best timing for treatment with chloroquine was the trophozoite stage (Chimanuka et al. 2001).

In contrast with previous reports (Saito-Ito et al. 2001), our results based on FCA data, showed that $\mathrm{IC}_{50}$ 's of each antimalarial compound were consistent with those obtained by microscopy, and ${ }^{3}[\mathrm{H}]$-hypoxanthine uptake.

In summary, despite hypoxanthine uptake is the gold standard method for testing in vitro antimalarial activity, our results confirm FCA as a better method to assess stage-specific antimalarial activity showing high reproducibility, automation of analysis, and efficient data processing. In contrast, hypoxanthine gives limited information on parasite stages and produces radioactive waste.

\section{ACKNOWLEDGEMENTS}

To Drs Juan De Sanctis for advice and support and Diana Ajami for manuscript review, and to Patricia Rodríguez, Eladia Paolini, and Angelyseb Dorta for assistance.

\section{REFERENCES}

Bard Y 1974. The Marquardt method. In Non Linear Parameter Estimation, Academic Press Inc., New York, p. 94-96.

Cambie G, Caillard V, Beaute-Laffite A, Ginsburg H, Chabaud A, Landau I 1991. Chronotherapy of malaria: identification of drug-sensitive stage of parasite and timing of drug delivery for improved therapy. Ann Parasitol Hum Comp 66: 14-26.

Chimanuka B, Francois G, Timperman G, Heyden YV, Holenz J, Plaizier-Vercammen J, Bringmann G 2001. A comparison of the stage-specific efficacy of chloroquine, artemether and dioncophylline $\mathrm{B}$ against the rodent malaria parasite Plasmodiun chabaudi chabaudi in vivo. Parasitol Res 87: 795-803.

Desjardins RE, Canfield CJ, Haynes JD, Chulay JD 1979. Quantitative assessment of antimalarial activity in vitro by a semiautomated microdilution technique. Antimicrob Agents Chemother 16: 710-718.

Domínguez J, Basante W, Charris J, Riggione F 1996. Synthesis and activity of some quinolone derivatives against Plasmodium falciparum in vitro. Il Farmaco 51: 407-412.

Dominguez JN, Charris J, Mendez B 1998. ${ }^{13}$ C NMR spectral characterization of some antimalarial tricyclic quinolone derivatives. Magn Reson Chem 36: 454-456.

Hooper DC, Wolfson JS 1985. The fluoroquinolones: pharmacology, clinical uses, and toxicities in humans. Antimicrob Agents Chemother 28: 716-721.

Janse CJ, van Vianen PH, Tanke HJ, Mons B, Ponnudurai T, Overdulve JP 1987. Plasmodium species: flow cytometry and microfluorometry assessments of DNA content and synthesis. Experimental Parasitol 64: 88-94.

Kumaratilake LM, Ferrante A 2000. Opsonization and phagocytosis of Plasmodium falciparum merozoites measured by flow cytometry. Clin Diag Lab Immunol 7: 9-13.

Lambros C, Vanderberg JP 1979. Synchronization of Plasmodium falciparum erythrocytic stages in culture. J Parasitol 65: 418-420.

Macreadie I, Ginsburg H, Sirawaraporn W, Tilley L 2000. Antimalarial drug development and new targets. Parasitol Today 16: 438-444.

Ouellette M, Kunding C 1997. Microbial multidrug resistance. 
Int J Antimicrob Agents 8: 179-187.

Pattanapanyasat K, Thaithong S, Kyle DE, Udomsangpetch R, Yongvanitchit K, Hider RC, Webster HK 1997. Flow cytometric assessment of hydroxy piridinone iron chelators on in vitro growth of drug-resistant malaria. Cytometry 27: 84-91.

Pattanapanyasat K, Udomsangpecth R, Webster HK 1993. Twocolor flow cytometric analysis of intraerythrocytic malaria parasite DNA and surface membrane-associated antigen in erythrocytes infected with Plasmodium falciparum. Cytometry 14: 449-454.

Pattanapanyasat K, Yongvanitchit K, Heppner DG, Tongtawe P, Kyle DE, Wepster HK 1996. Culture of malaria parasites in two different red blood cell populations using biotin and flow cytometry. Cytometry 25: 287-294.

Pattanapanyasat K, Yongvanitchitk K, Tongtawe P, Tachavanich K, Wanachiwanawin W, Fucharoen S, Walsh DS 1999. Impairment of Plasmodium falciparum growth in thalassemic red blood cells: further evidence by using biotin labeling and flow cytometry. Blood 93: 3116-3119.

Posner G, González L, Cumming J, Klinedinst D, Shapiro T 1997. Synthesis and antimalarial activity of heteroatoms containing bicyclic endoperoxides. Tetrahedron 53: 37-50

Rosenthal PJ, Olson JE, Lee GK, Plamer JT, Klaus JL, Rasnick
D 1996. Antimalarial effects of vinyl sulfone cysteine proteinase inhibitors. Antimicrob Agents Chemother 40: 16001603.

Saito-Ito A, Akai Y, Shenyi H, Kimura M, Kawabata M 2001. A rapid, simple and sensitive flow cytometric system for detection of Plasmodium falciparum. Parasitol Int 50: 249257.

Trager W, Jensen JB 1976. Human malaria parasite in continuous culture. Science 193: 674-675.

Tripathi KD, Sharma AK, Valecha N, Biswas S 1993. In vitro activity of fluoroquinolones against chloroquine-sensitive and chloroquine-resistant Plasmodium falciparum. Indian J Malariol 30: 67-73.

van Vianen PH, Thaithong S, Reinders PP, van Engen A, van der Keur M, Tanke HJ, van der Kaay HJ, Mons B 1990. Automated flow cytometric analysis of drug susceptibility of malaria parasites. Am J Trop Med Hyg 43: 602-607.

Vindelov L 1977. Flow microfluorometric analysis of nuclear DNA in cells from solid tumors and cell suspensions. A new method for rapid isolation and staining of nuclei. Virchows Arch B Cell Pathol 24: 227-242

Yayon A, Vande Waa JA, Yayon M, Geary TG, Jensen JB 1983. Stage-dependent effect of chloroquine on Plasmodium falciparum in vitro. J Protozol 30: 642-647. 